\title{
Fabrication of novel porous membrane from biobased water- soluble polymer (hydroxypropylcellulose)
}

\author{
A. Hanafia ${ }^{1}$, C. Faur ${ }^{1}$, A. Deratani ${ }^{1}$, P. Guenoun ${ }^{2}$, H. Garate ${ }^{2}$, D. Quemener ${ }^{1}$, C. Pochat-Bohatier ${ }^{1}$, D. \\ Bouyer*1 \\ *corresponding author: denis.bouyer@umontpellier.fr \\ ${ }^{1}$ IEM (Institut Européen des Membranes), UMR5635 (CNRS-ENSCM-UM), Université de Montpellier, \\ Place E. Bataillon, F-34095 Montpellier, France \\ ${ }^{2}$ CEA Saclay, LIONS, NIMBE, CEA, CNRS, Université Paris-Saclay, CEA Saclay, France
}

\section{Abstract}

Herein, a novel polymeric porous membrane was developed, without the use of any organic solvent in the initial dope solution and using a biobased polymer derived from cellulose: Hydroxypropylcellulose (HPC). HPC was dissolved in water (20 wt\%) and the phase separation was induced by increasing the temperature above the Lower Critical Solution Temperature (LCST) of the polymer solution, around $40^{\circ} \mathrm{C}$ in the concentration range concerned in this study. To fix the membrane morphology and to prevent any resolubilization in water during filtration tests, a chemical crosslinking was performed using Glutaraldehyde. The phase diagram of HPC/water system was first studied and not only the cloud point but also the spinodal curves were determined using optical transmission techniques. It was exhibited that HPC phase diagram is very weakly dependent on concentration up to large concentrations and that the metastable region is very small, i.e. the cloud point and the spinodal curves are very close in a large range of concentration. The membrane stability was tested in water and some organic solvents, thus demonstrating the efficiency of the chemical crosslinking during membrane formation. The swelling and mechanical properties of HPC membranes were also investigated depending on the operating condition during membrane formation, showing that the temperature ramp during the membrane formation, from initial to final 
temperature, have a significant effect on the crosslinking efficiency and hence on the swelling properties. Finally, adding a porogen (PEG200) into the collodion, filtration tests were performed and exhibited that the membrane filtration properties depend on the temperature ramp as well, showing higher water flux for the highest temperature ramp tested.

\section{Keywords}

Membranes, green chemistry, biobased polymer, water-soluble polymer

\section{Introduction}

Membrane separation technologies are nowadays extensively used in many engineering applications including water treatment and purification. Polymeric membrane materials account for a large majority (ca $85 \%$ ) of the membrane material demand. Within the polymeric membrane segment, synthetic polymers including polysulfone, polyimide, nylon, polyacrylonitrile, PVDF and others dominate the market. Polymer phase separation, which consists of lowering the solvency of the solvent to the polymer, is mostly used in manufacturing of such porous materials: a dope solution, i.e. a polymer dissolved in a solvent, is cast as a film on a support or extruded as a hollow fiber. Subsequently, a change in the thermodynamic state disturbs the solution equilibrium. Thermally Induced Phase Separation (TIPS) and Non-solvent Induced Phase Separation (NIPS) are the most convenient and common methods for manufacturing porous polymeric membranes. Many membrane structures can be formed depending on operating conditions (process parameters) and system properties (formulation) [1-3]. NIPS process (including immersion precipitation and Vapour Induced Phase Separation, VIPS) consists of adding a non-solvent into a binary polymer/solvent solution. TIPS process is classically conducted by cooling a hot polymer/solvent solution, inducing thermodynamic instability and thereby phase separation [4-11]. Porous membranes are thus produced by phase separation from polymer solutions and from economic point of view, water is generally used as the non-solvent. Since the polymer concentration is usually in the range $15-20 \%$ 
and since coagulation and washing baths require being often renewed, large amounts of aqueous solutions must be treated. For instance, $10 \mathrm{~m}^{2}$ of ultrafiltration membrane need about 1 to $1.5 \mathrm{~kg}$ of solvent. The major fraction can theoretically be recycled after an expensive distillation stage (ca $90 \%$ for slightly volatile solvents), a fraction is lost by evaporation in air (not negligible for highly volatile solvents and high temperature process) and the last fraction is sent for wastewater treatment. Moreover, the use of organic solvent in the membrane formation has consequences on industrial fabrication units, which must be adapted to protect workers from solvent toxicity. Therefore, the use of organic solvents has a significant cost input in the process of membrane manufacturing apart the environmental and public health impact. Facing the challenges of decreasing these impacts, shift of paradigm is needed from conventional solvents towards greener ones including bio-based solvents, ionic liquids and supercritical carbon dioxide [12-13]. Among them, water is obviously the safer and the less demanding on energy. Nevertheless, to the best of our knowledge, very few papers have been devoted to the membrane preparation in water [14-16].

In this respect, the present work aims at preparing polymeric membranes using water as the solvent. This objective clearly addresses the issue of designing greener processes avoiding the use of organic solvents, one of the cornerstones of the green chemistry area. The other objective is to design membrane from bio-sourced materials. The economy of atoms and reduction of energy in the manufacturing of membranes is assumed to be achieved in a friendly environmentally approach using (semi)natural and water-soluble polymers. Cellulose ethers fulfill these criteria. These derivatives are characterized by a lower critical solution temperature (LCST) that can be used to induce the phase separation. The polymer phase separates through a temperature increase above the LCST [17], calling the process LCST-TIPS in contrast to the commonly used upper critical solution temperature (UCST)-TIPS for membrane preparation with synthetic polymers. Phase separation with LCST systems can usually be tuned at moderate, close to ambient temperatures, limiting energy consumption. The available literature on the LCST-TIPS process is very limited. Qin and coworkers prepared membrane from polyethersulfone/poly(vinyl pyrrolidone) $\mathrm{K} 90 / \mathrm{N}, \mathrm{N}$-dimethylacetamide 
(DMA)/1,2-propanediol (1,2-PD) system [18-19] and cellulose acetate/poly (vinyl pyrrolidone) 360K/N-methyl-2-pyrrolidone (NMP)/1,2-propanediol system [20]. Recently, free organic solvent membranes have been prepared from PVA/water system [16]

Hydroxypropylcellulose (HPC), non-ionic ether of native cellulose, is a polymer with temperature dependent water solubility. Thanks to its renewable origin, ease of production, non-toxicity and optical properties, HPC is widely used (i) for the preparation of dosage drugs forms and coatings, (ii) as viscosity modifier in many product formulations [21]. Furthermore HPC is studied in polymer physics for its lyotropic liquid crystalline phase behavior [21]. HPC has also been largely studied to develop hydrogels for controlled release of active substances above its LCST [22-24].

In this study, porous membranes prepared by LCST-TIPS process from HPC will be demonstrated. The dynamics of membrane formation, including the coupling between the phase inversion dynamics, the crosslinking and the mass transfer by evaporation will be discussed. Since this polymer was poorly studied in the past, the phase diagram was investigated, including both the determination of the cloud point curve and the spinodal curve using light scattering techniques. The membrane morphology of HPC membranes was reported and discussed as a function of the operating parameters including the initial formulation and the temperature ramp. The stability and the mechanical properties of the membranes were then investigated. Finally, preliminary filtration tests in water will be presented.

\section{Experimental}

\subsection{Materials}

Hydroxypropylcellulose (HPC) was obtained from Sigma Aldrich (Fig. 1). Its average molecular weight, determined by size-exclusion chromatography, was $M w 80,000 \mathrm{~g} / \mathrm{mol}$. Distilled water was used as solvent in polymer/solvent system. The washing bath was exclusively composed of distilled water. Glutaraldehyde (GA solution $25 \mathrm{wt} \%$ ) was chosen as the crosslinking agent, hydrochloric acid ( $\mathrm{HCl}$ 
solution $37 \mathrm{wt} \%)$ as the crosslinking reaction catalyst and polyethylene glycol $M w 200 \mathrm{~g} / \mathrm{mol}$ as porogen (PEG200). All of these products were purchased from Sigma Aldrich and all the reagents were used without further purification.

\subsection{Methods}

\subsubsection{Preparation of polymer solutions}

Hydroxypropylcellulose (HPC) aqueous solution was prepared by dispersing HPC powder in distilled water preheated at $60{ }^{\circ} \mathrm{C}$ under magnetic stirring. The initial HPC concentration in water was $20 \mathrm{wt} \%$. The dispersion was then cooled to ambient temperature to promoting polymer dissolution and obtaining homogeneous polymer solution. A given amount of additives was then blended into the solution under magnetic stirring. The solution prepared was degassed under static conditions at $4{ }^{\circ} \mathrm{C}$ overnight. The polymer concentration (20 wt\%) was chosen in order to reach a convenient viscosity for casting the solution onto the glass plate (the viscosity of HPC solutions was equal to 18.98 Pa.s during casting) and to have a moderate cloud point temperature (close to $40{ }^{\circ} \mathrm{C}$ ). The chemical crosslinker, Glutaraldehyde, was added in the collodion, at a concentration of 0.5 wt $\%$ (versus the total solution weight). The catalyzer, $\mathrm{HCl}$, was added just before casting the polymer solution onto the heating plate. $\mathrm{HCl}$ concentration in the collodion was equal to $1 \mathrm{wt} \%$. The composition of the polymer solution (see Table 1) was fixed from previous study of Suto et al. [32] to optimize the crosslinking, i.e. to obtain an isotropic membrane with well distributed chemical bonds in the whole membrane: Glutaraldehyde weight ratio $1 / 4$ (GA/HPC) was shown to be the most efficient ratio for obtaining a stable polymer matrix. The concentration of each component in the collodion was summarized in Table 1.

Table 1. Concentration of polymer and additives in the collodion.

\begin{tabular}{|l|l|l|l|}
\hline HPC & GA & HCl & PEG200 \\
\hline 20wt\% & 0.5 wt\% & 1 wt\% & 5 wt\% \\
\hline
\end{tabular}




\subsubsection{Cloud point measurements}

Polymer solutions were prepared by weighing at different concentrations and put after thorough mixing and heating/cooling steps in quartz cuvettes. These cuvettes were immersed in a water thermostated bath at a temperature in the one-phase region. The temperature was then progressively increased by steps of $0.5^{\circ} \mathrm{C}$ per 30 minutes. A laser beam is sent through the cuvette and the transmission of the beam is measured as a function of temperature by means of two photodiodes, one as a reference of the incident beam and the second on the transmitted path.

\subsubsection{Membranes preparation}

The blend solution was cast onto a glass plate at room temperature with an automatic microcoater (Erichsen Instuments) using two speed casting: $3.4 \pm 0,2 \mathrm{~cm} / \mathrm{s}$ and $15.0 \pm 0,5 \mathrm{~cm} / \mathrm{s}$. The thickness of the solution was fixed at $250 \mu \mathrm{m}$ for the whole experiments. The sample was then placed on a Linkam hotstage LTS 420, which was chosen for its accurate thermal control and its built-in stage manipulator; the software Linksys 32 allowed to perfectly control the temperature ramp (from 0.01 to $\left.120^{\circ} \mathrm{C} / \mathrm{min}\right)$ and the target temperature $\left(60^{\circ} \mathrm{C}\right)$; two temperature ramps were performed in this study to increase temperature from ambient to target temperature: $2^{\circ} \mathrm{C} / \mathrm{min}$ and $120^{\circ} \mathrm{C} / \mathrm{min}$. The membrane formation was conducted at ambient atmosphere, i.e. under $\mathrm{RH}$ close to $50 \%$. HPC concentration was chosen in such a way that the solution viscosity of HPC/water system (close to 7 Pa.s without additives) allowed (i) easy handling of the solution and additives admixture with a fluent homogenization, (ii) casting the polymer solution onto a glass plate for flat membranes. The whole operating conditions were reported in Table 2 .

The membrane was then removed from the glass plate and placed in a washing bath containing distilled water in order to remove excess of additives agents. The membrane was stored in distilled water prior to further water flux measurements, or oven dried at $30^{\circ} \mathrm{C}$ for further morphological and mechanical characterizations.

Table 2. Operating conditions for membrane preparation. 


\begin{tabular}{|l|l|l|l|l|l|l|}
\hline Initial film & \multicolumn{2}{l|}{ Casting speed } & \multicolumn{2}{l|}{ Temperature ramp } & RH & Water \\
thickness & \multicolumn{2}{l|}{} & & temperature \\
\hline $250 \mu \mathrm{m}$ & $15.0 \pm 0,5 \mathrm{~cm} / \mathrm{s}$ & $3.4 \pm 0,2 \mathrm{~cm} / \mathrm{s}$ & $2{ }^{\circ} \mathrm{C} / \mathrm{min}$ & $120^{\circ} \mathrm{C} / \mathrm{min}$ & $50 \%$ & $20^{\circ} \mathrm{C}$ \\
\hline
\end{tabular}

\subsubsection{Characterization of the membranes}

\subsubsection{Scanning electron microscopy}

The SEM micrographs were produced using HITACHI S-4800 equipped with a Sputler Coater SC 7620 for sample metallization. The HPC films were fractured in liquid nitrogen; the samples surface and cross section were analyzed by SEM.

\subsubsection{Viscosity}

The viscosity of HPC solutions (with and without additives) has been measured using a Rheometric Scientific (Physica MCR 301, Anton Paar) equipped with a parallel-plate geometry (air gap between 0.01 and $0.1 \mathrm{~mm}$ ). All the experiments have been performed at $25^{\circ} \mathrm{C}$.

\subsubsection{Crosslinking}

The crosslinking was followed using the same Rheometric Scientific (Physica MCR 301, Anton Paar) equipped with a parallel-plate geometry; variable oscillation amplitude and variable oscillation frequency were tested to determine the best operating conditions (linear domain) and to fix the operating conditions, i.e. shear stress and oscillatory frequency. The variation of the elastic $\left(G^{\prime}\right)$ and viscous $\left(G^{\prime \prime}\right)$ modulus were followed under small amplitude oscillatory shear stress (1\%) and $1 \mathrm{~Hz}$ frequency. The rheological measurements were conducted in closed system (the polymer solution was put between two glass plates and isolated from the external environment) to prevent solvent evaporation during experiments.

\subsubsection{Mechanical properties}

The measurements of mechanical properties were carried out under dry conditions on the Rheometric Scientific (Physica MCR 301, Anton Paar) testing machine at $25^{\circ} \mathrm{C}$ with a frequency of 
$1 \mathrm{~Hz}$. A film sample was cut into pieces with $4 \times 1 \mathrm{~cm}^{2}$. The sample thickness was determined with a digital micrometer (MITUTOYO with an accuracy of $0.001 \mathrm{~mm}$ ). Five measurements were performed on each membrane at different locations. The sample was attached to the tensile grips and the test was finished once the sample broke. Three successful measurements were used to obtain average values for mechanical properties. The stress $\sigma$ was determined in terms of the original cross-sectional area, and the strain defined as $\varepsilon=\left(I-I_{0}\right) / I_{0}$, where $I$ and $I_{0}$ are the length at the time of data collection and original length, respectively.

\subsubsection{Solubility and water sorption}

A sample piece $\left(2 \times 1 \mathrm{~cm}^{2}\right)$ was cut from the HPC film oven dried at $30{ }^{\circ} \mathrm{C}$ for $24 \mathrm{~h}$ and immersed in distilled water for 40 days. The swelling ratio was determined at ambient temperature, based on the following equation: $S R(\%)=\left(W-W_{0}\right) / W_{0} \times 100$, where $W_{0}$ is the initial weight of dried sample piece and $W$ is the weight of swollen sample piece (the surfaces of the film were wiped with soft paper tissue before measurements).

\subsubsection{Water flux measurements}

Ultra-pure water was used to characterize the pure water flux through porous HPC membranes at temperature $25 \pm 0.5^{\circ} \mathrm{C}$ in a stirred ultrafiltration cell (Millipore). The effective membrane area was $4.1 \mathrm{~cm}^{2}$ and the membrane was supported by a non-woven material (Polyethylene Terephthalate) to enhance the mechanical properties of HPC films. The pressure was gradually increased from 0 to 3.5 bars with pressure steps of 0.2 bar during $15 \mathrm{~min}$. Each membrane was conditioned prior to the filtration tests, i.e. the pressure was gradually increased from 0.1 to 3.5 bars (pressure step of 0.2 bar during $30 \mathrm{~min}$ ). 


\section{Results and Discussion}

\subsection{Phase diagram}

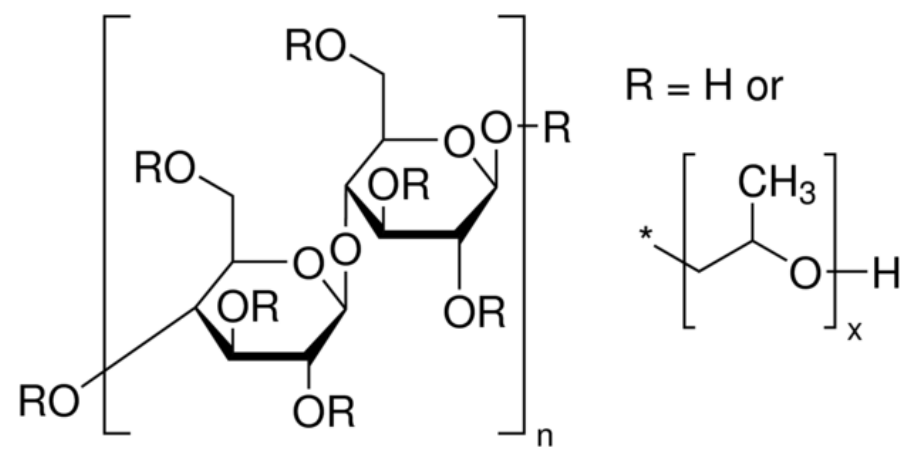

Figure 1. Chemical structure of $\mathrm{HPC}\left(\mathrm{R}=-\mathrm{H} /-\mathrm{CH}_{2} \mathrm{CH}-\left(\mathrm{OR}^{\prime}\right) \mathrm{CH}_{3}\right.$ with $\left.\mathrm{R}^{\prime}=-\mathrm{H} /-\mathrm{CH}_{2} \mathrm{CH}(\mathrm{OR}) \mathrm{CH}_{3}\right)$

The usual method found in literature for determining the cloud point temperature $\left(T_{C P}\right)$ of water soluble polymers solutions amounts to measuring the optical transmission through a sample. As the transition is usually spanning a broad interval of a few degrees a (rather arbitrary) method has been

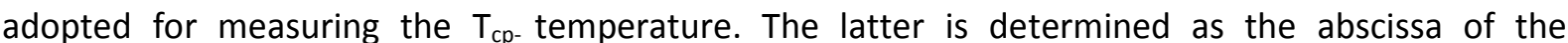
intersection of two limiting slopes of the transmission curve, one horizontal slope drawn at temperatures lower than the transition and one inclined slope representing the transmission decrease through the temperature interval (the principle is depicted in T. Kyu, P. Zhuang, P. Mukherjee [25] for the scattering intensity and by E. Marsano and G. Fossati [26] for the transmission). Our measurement is reported in Fig.2 [27-28]. 


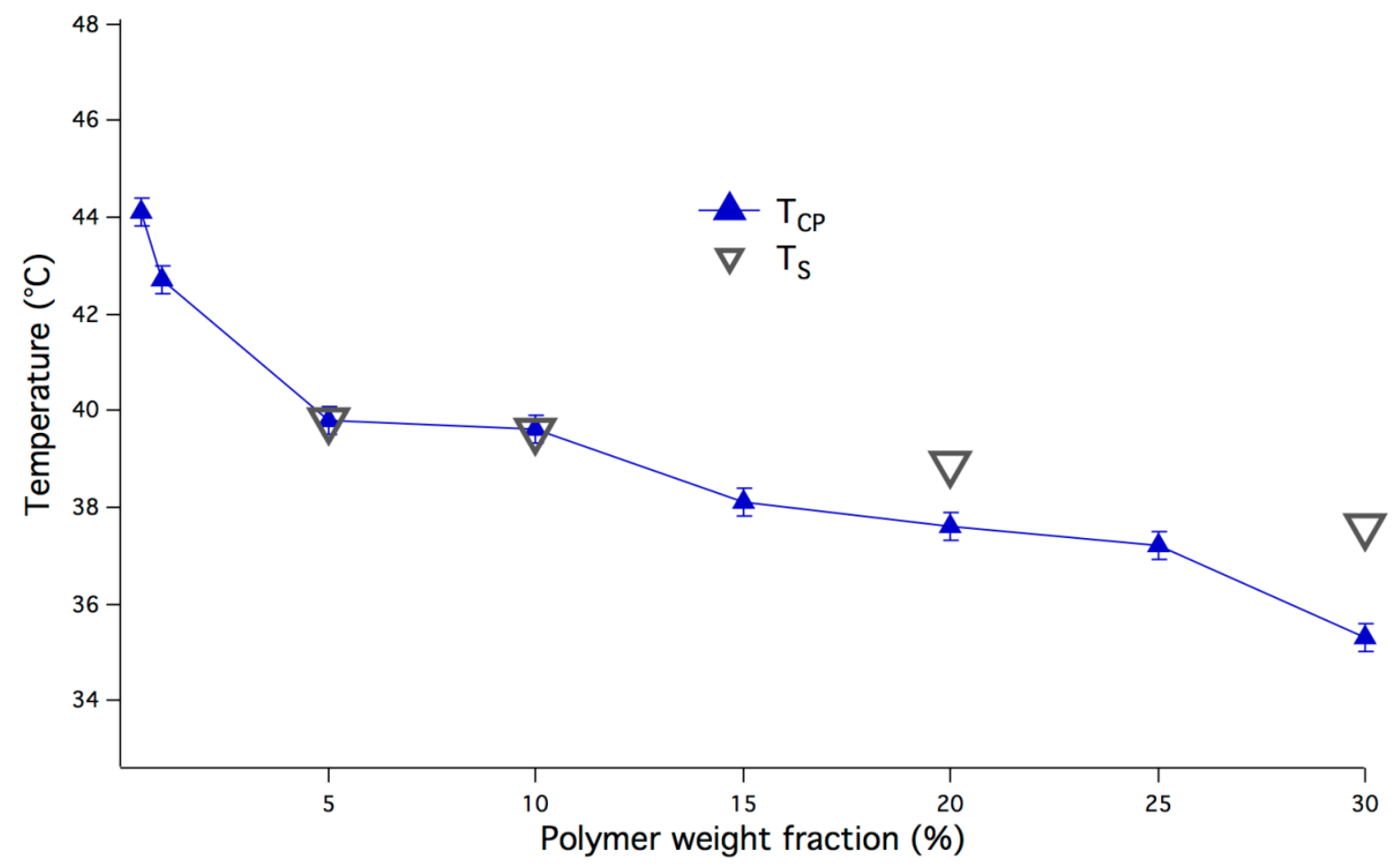

Figure 2. Cloud point temperatures and spinodal temperatures measured as described in text

The spinodal temperature, defined as the temperature at which the scattered light intensity extrapolated at zero angle (meaning zero wavevector of transfer) diverges, was also measured (Fig. 2). The values of $T_{c p}$ are in good agreement with the previously reported ones for dilute samples by $F$. Winnik [28] and for concentrated samples by T. Kyu and P. Mukherjee [29], and E. Marsano and G. Fossati [26]. The spinodal temperature is, to our knowledge, reported here for the first time [30-32]. The place where both temperatures coincide should indicate the location of the critical point (between 5 and 10 wt\%) which is shifted to higher concentrations with respect to a monodisperse sample of same $M_{w}$ [33]. It must be noted, that contrary to pure polydisperse phase diagrams originating from a Flory-Huggins-like modeling, HPC phase diagram is very weakly dependent on concentration up to large concentrations like is PNIPAm phase diagram [34]. 


\subsection{Fabrication of HPC membranes and morphological structure characterization}

\subsubsection{Simultaneous phenomena involved in the membrane formation mechanisms}

The membrane preparation is represented in Fig. 3: once the polymer was dissolved in water at ambient temperature, the polymer solution was cast onto a glass plate at two casting speeds and then placed onto a heating plate. Both the target temperature and the temperature ramp could be modified during the heating of the polymer solution for inducing the phase inversion. At a temperature higher than the cloud point temperature of the HPC/water system, three phenomena occurred concomitantly: phase separation, crosslinking and water evaporation. At the end of this stage, the temperature was reduced until ambient temperature and the membrane was washed in a water bath before storage.

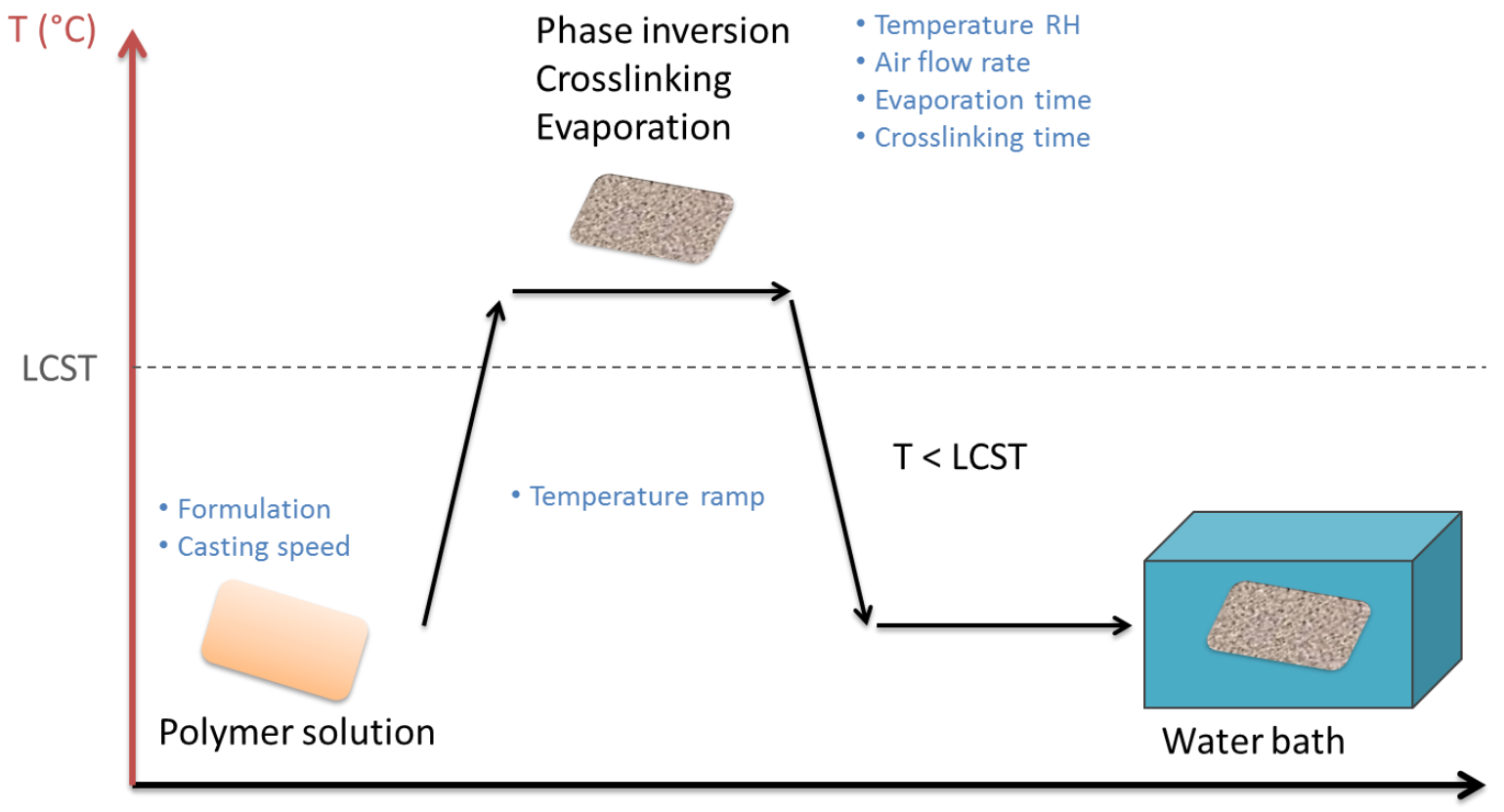

Time

Figure 3. Schematic of membrane preparation

Polymeric membranes made from HPC/water system were prepared using LCST-TIPS method and a chemical crosslinking was performed using Glutaraldehyde (GA). The chemical crosslinking aimed at (i) preventing the membrane dissolution in water solution in filtration operation and (ii) fixing the membrane morphology to obtain a porous structure. As aforementioned, a PEG200 was added to the 
formulation as porogen agent. The chemical crosslinking was performed concomitantly with the phase separation, i.e. the crosslinking agent was added in the initial formulation just before casting the solution onto the glass plate. The temperature ramp was carefully controlled during membrane formation and solvent evaporation occurred since the system was open to air; three phenomena were involved in the membrane formation mechanisms: phase inversion, crosslinking and solvent evaporation. Each phenomenon was affected by both others: for instance, the crosslinking could have an influence on phase inversion dynamics by reducing the polymer chain mobility; in the same time, the evaporation could affect the crosslinking due to the progressive increase of the polymer concentration, and phase inversion and the solvent evaporation are also interdependent: on the one hand, evaporation during phase inversion leads to a deviation of the composition path in the phase diagram and on the other hand, the temporal variation of the lean composition phase due to phase separation dynamics affects the evaporation dynamics. To start deciphering this complex interplay, two temperature ramps were tested $\left(2\right.$ and $\left.120^{\circ} \mathrm{C} / \mathrm{min}\right)$, leading to different membrane formation dynamics in terms of solvent evaporation, crosslinking rate and phase separation dynamics.

\subsubsection{Crosslinking}

- Closed system (without solvent evaporation)

Crosslinking dynamics was first followed in closed system using a rheometer (Physica MCR 301, Anton Paar). In these conditions, water evaporation was not possible and hence the polymer concentration was kept constant during the experiments. The tests were performed under small amplitude oscillatory shear stress (1\%) and a frequency of $1 \mathrm{~Hz}$. Surprisingly, the elastic modulus $\mathrm{G}^{\prime}$ did not cross the viscous modulus $\mathrm{G}^{\prime \prime}$, even after 16 hours of experiments despite the presence of the crosslinking agent and the catalyzer in appropriate proportions. This result exhibited that the crosslinking was not efficient enough in such operating conditions, i.e. the number of bonds between polymer chains was too low for inducing a sol-gel transition. Indeed, the creation of bonds between HPC polymer chains was hindered because of HPC chemical structure: due to the random substitution of - $\mathrm{OH}$ groups by -OR groups (Fig. 1), at a polymer concentration of $20 \mathrm{wt} \%$ and using 
GA, the probability to have a favorable conformation for chemical crosslinking was quite low, thus explaining the low crosslinking efficiency in such closed system.

- Open system (with solvent evaporation)

During the membrane preparation, the system was open to atmosphere and solvent evaporation occurred simultaneously to phase inversion and crosslinking. In this case, the polymer concentration progressively increased over time until the membrane structure was fixed (after almost complete water evaporation). The crosslinking efficiency was characterized by checking the membrane stability after its immersion in water during several days. Swelling and re-solubilization ratios were determined for the two different temperature ramps: 2 and $120^{\circ} \mathrm{C} / \mathrm{min}$. For those two conditions of temperature ramp, we demonstrated that HPC membrane did not dissolve in water after 40 days of immersion (the re-solubilization was null). Such results obtained in water bath clearly demonstrated the efficiency of the chemical crosslinking reaction performed during membrane formation. The same test was then determined in two aprotic solvents (dimethylsulfoxide and tetrahydrofurane), and two protic solvents (methanol and chloroform). In all case too, no re-solubilization was observed, hence demonstrating that the crosslinking was efficient enough to prevent the dissolution in polar aprotic solvents as well.

\subsubsection{Membrane morphologies}

Classically, the membranes formed by UCST-TIPS process exhibit symmetric morphologies since the heat transfer induced by a temperature quench is fast comparing to the mass transfer, which would create anisotropy in the membrane structure; Indeed it has been previously demonstrated that anisotropic structures could be formed if an evaporation stage is done prior to TIPS process [8-11]. For this work, the SEM photographs of the final membranes prepared by LCST-TIPS method were reported in Fig. 4. 
Top surface

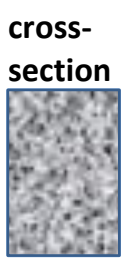

Top cross-

section

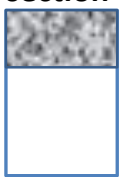

Middle

cross-

section

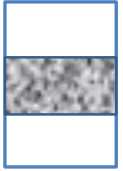

Low cross-

section

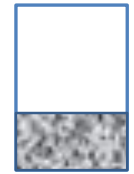

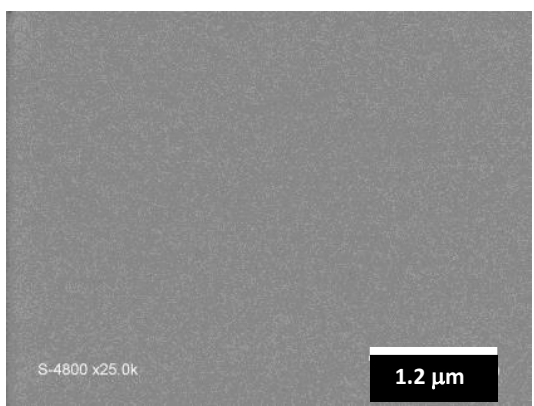

(a)

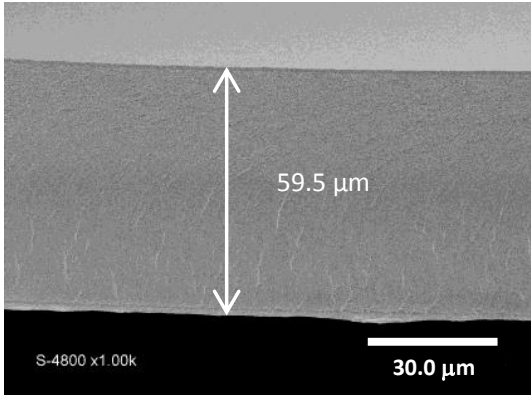

(b)

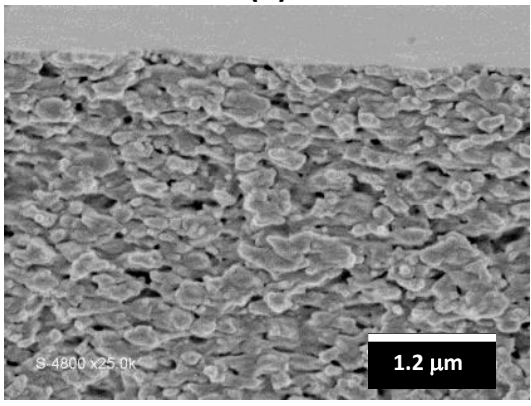

(c)

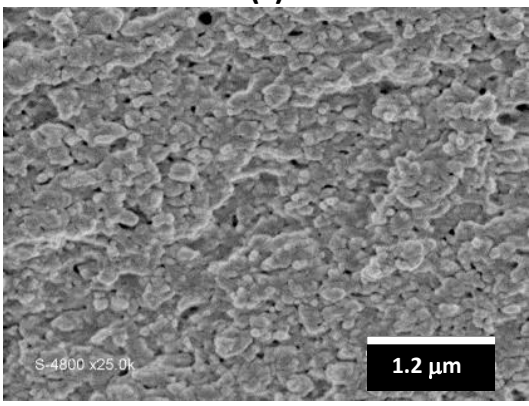

(d)

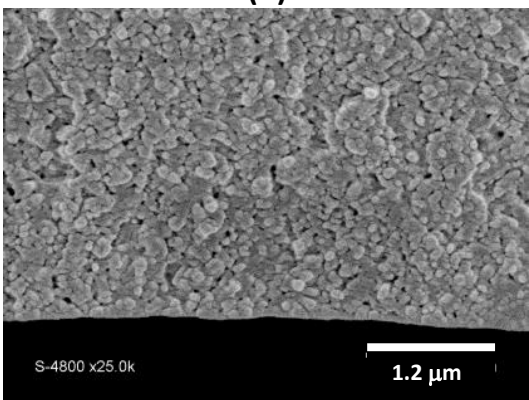

(e)

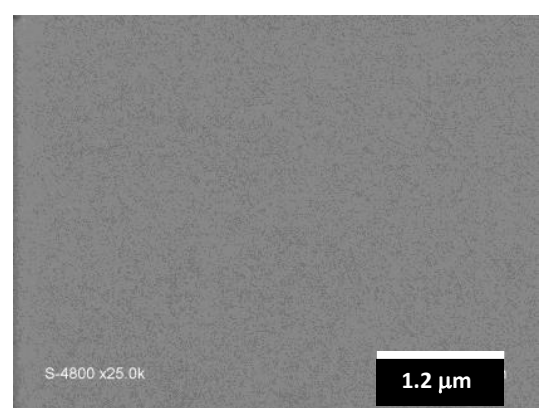

(g)

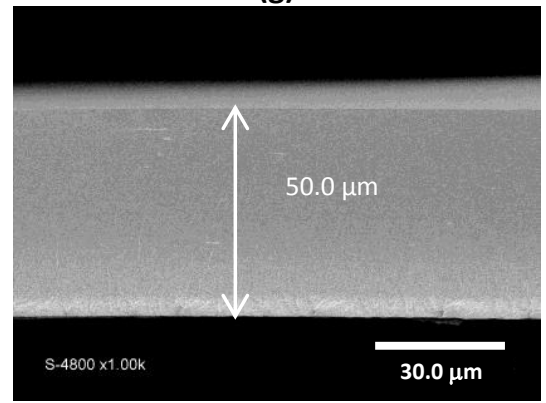

(h)

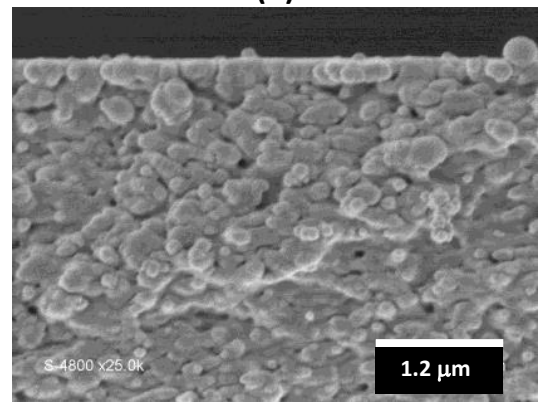

(i)

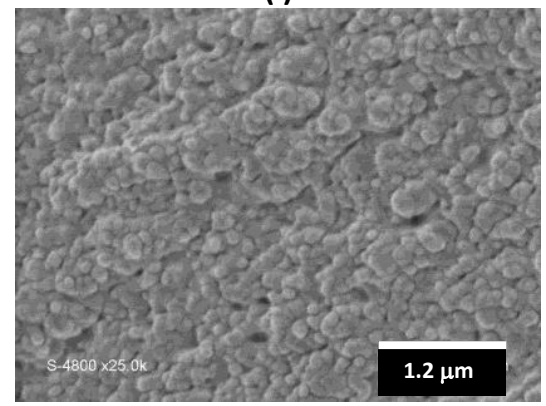

(j)

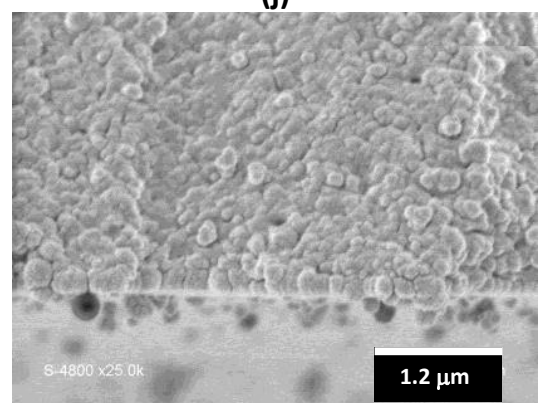

(k) 


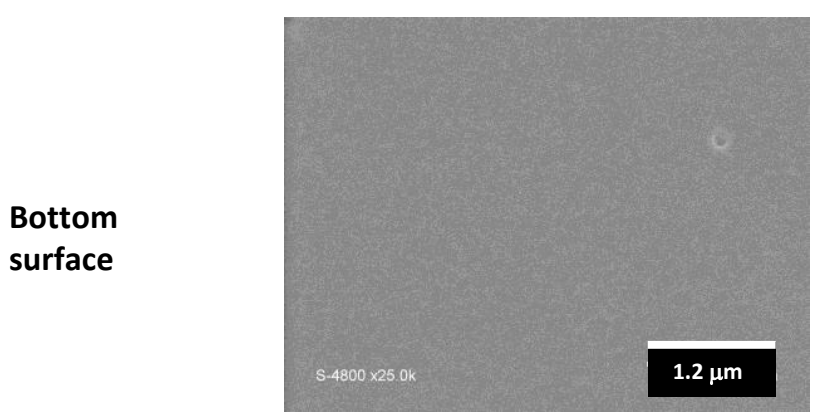

(f)

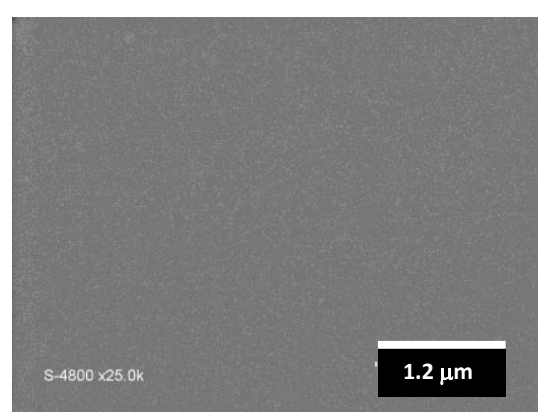

(I)

Figure 4. Cross-sections of HPC/GA/PEG200 membranes prepared using two temperature ramps

The membranes were prepared using the procedure described in the material and methods section and the formulation was the same as the one chosen for the crosslinking experiments: HPC $20 \mathrm{wt} \%$, GA 5 wt $\%, \mathrm{HCl} 1 \mathrm{wt} \%, \mathrm{PEG} 2005 \mathrm{wt} \%$. For a temperature ramp of $120^{\circ} \mathrm{C} / \mathrm{min}$, Figures $4 \mathrm{a}$ and $4 \mathrm{f}$ present the SEM of top and bottom surfaces, respectively, and Figures $4 b, 4 c, 4 d$ and 4 e present the SEM of cross-sections at different locations in the membrane thickness. For a temperature ramp of $2{ }^{\circ} \mathrm{C} / \mathrm{min}$, Figures $4 \mathrm{~g}$ and $4 \mathrm{I}$ present the SEM of top and bottom surfaces, respectively, and Figures $4 \mathrm{~h}$, $4 i, 4 j$ and $4 k$ present the SEM of cross-sections. The top and bottom surfaces of the membranes seem to show a very low apparent porosity, whatever the temperature ramp, meaning that low pore sizes can be expected in both operating conditions. These photographs exhibit that the final membrane thickness in dry state after solvent evaporation is close to $50 \mu \mathrm{m}$ whatever the temperature ramp, i.e. close to $20 \%$ of the initial thickness $(250 \mu \mathrm{m})$. This indicates that the global porosity of the HPC membrane is quite low. Nevertheless, zooms performed near the top surface (Figures $4 \mathrm{c}$ and $4 \mathrm{i}$ ), at the middle (Figures $4 d$ and $4 j$ ) and at the bottom (Figures $4 e$ and $4 k$ ) of the membrane show that HPC membranes are porous in the whole thickness, for both temperature ramps. SEM observations in Fig. 4 suggest that the phase separation led to the formation and the coexistence of two interconnected phases, a rich phase composed of concentrated HPC and a lean phase mainly composed of water and GA. This polymeric structure was bicontinuous, reminiscent of spinodal decomposition mechanism; this result was expected for two main reasons: (i) as shown by the phase diagram determined in this work and reported in Fig. 2, the spinodal and binodal curves were shown to be very close, and hence the metastable region is very thin for the HPC/water system. 
Furthermore, (ii) the target temperature was significantly higher than the spinodal curve at given polymer concentration, and hence whatever the temperature ramp, the system did not stay enough time in the metastable region to induce nucleation and growth mechanism that would have formed cellular structures in the membrane.

Fig. 4 also shows that although the HPC membrane seemed to be isotropic regarding the global cross-section photographs (Fig. $4 \mathrm{~b}$ and $4 \mathrm{~h}$ ), the zooms at different locations in the membrane thickness exhibit anisotropy between the top and the bottom cross-section. More precisely, the porosity is higher near the upper interface (air/membrane interface) than below in the membrane thickness, and the more dense structure can be observed just at the middle cross-section. This observation was done whatever the temperature ramp, i.e. 2 or $120^{\circ} \mathrm{C} / \mathrm{min}$, but the morphologies obtained at these different temperature ramps are different: the membrane porosity is lower when the membranes were prepared at $2{ }^{\circ} \mathrm{C} / \mathrm{min}$ comparing to those prepared at $120^{\circ} \mathrm{C} / \mathrm{min}$. A difference of evaporation rate combined to different cross-linking efficiencies between 2 and $120^{\circ} \mathrm{C} / \mathrm{min}$ would be probably the reason that explains those differences. Indeed, since the cross-linking was performed by a chemical reaction between glutaraldehyde and HPC polymer chains, its efficiency directly depends on the temperature, and as a consequence also on the temperature ramp because all phenomena involved in the membrane structuration are coupled. Faster the temperature will be reached during membrane formation, earlier the membrane structure will be fixed by chemical crosslinking. At $120^{\circ} \mathrm{C} / \mathrm{min}$, the target temperature is reached after only $20 \mathrm{~s}$ (from $20^{\circ} \mathrm{C}$ to $60{ }^{\circ} \mathrm{C}$ ) whereas it is reached after $20 \mathrm{~min}$ at $2{ }^{\circ} \mathrm{C} / \mathrm{min}$. At the lowest temperature ramp, the evaporation is therefore expected to be almost finished when the membrane structure is fixed and most of the water leaved the system. In this case, the polymer chains keep on moving and collapse during water extraction, thus forming the dense-like structure presented in Fig. $4 \mathrm{i}, 4 \mathrm{j}$, and $4 \mathrm{k}$. On the contrary, at $120^{\circ} \mathrm{C} / \mathrm{min}$, the morphology is fixed much earlier by cross-linking and hence the morphology is fixed whereas the water extraction by evaporation is not finished, thus keeping higher porosity in the membrane structure. 
Finally, SEM photographs were obtained at different concentrations of PEG200 (from 0 to 5 wt\%), which exhibited that the membrane morphology was not affected by the formulation (figure not shown in the paper).

\subsubsection{Water sorption}

Water sorption experiments were performed on HPC membranes with and without PEG200 in the initial formulation. The membranes were prepared at the two aforementioned temperature ramps: the membranes were stored during 40 days after their preparation in a water bath at ambient temperature (Table 3). In Table 3, the water sorption is expressed in swelling ratio (SR) defined in section 2.2.4.5.

Table 3. Swelling ratio (SR) of HPC membrane after 40 days storage in water bath.

\begin{tabular}{|c|c|c|}
\cline { 2 - 3 } \multicolumn{1}{c|}{} & $120^{\circ} \mathrm{C} / \mathrm{min}$ & $2^{\circ} \mathrm{C} / \mathrm{min}$ \\
\hline HPC/GA & $45 \% \pm 4 \%$ & $87 \% \pm 8 \%$ \\
\hline HPC/GA/PEG & $150 \% \pm 13 \%$ & $210 \% \pm 19 \%$ \\
\hline
\end{tabular}

The values of water sorption not only depend on the formulation, and more precisely the presence of PEG200, but also on the temperature ramp imposed during the membrane preparation by LCST-TIPS method. The table 1 exhibits that the water sorption was three times higher when the PEG200 was added in the initial formulation, going from $45 \%$ to $150 \%$, meaning that the porogen enhances hydrophilicity of the polymeric matrix, thus promoting the water intake. The great difference of water sorption observed depending on the formulation demonstrated that a significant proportion of porogen remained in the membrane after washing. Hydrophilic polymers are known to favor the water intake but the water sorption strongly depends on the cross-linking efficiency as well. Table 1 points out that the chemical cross-linking by GA was clearly more efficient at the highest temperature ramp; indeed the water sorption was divided by two without porogen between 
$2{ }^{\circ} \mathrm{C} / \mathrm{min}$ and $120^{\circ} \mathrm{C} / \mathrm{min}$ and by almost 1.5 when the PEG200 was added to the initial formulation, from $210 \%$ to $150 \%$.

Fig. 4. exhibited that the membrane morphology was different depending on the temperature ramp: the structure was denser, i.e. the porosity was lower, in the middle of the membrane thickness at the lowest temperature ramp $2^{\circ} \mathrm{C} / \mathrm{min}$. In the same time, the water sorption was shown to be much higher at this temperature ramp, indicating that the membrane was almost a hydrogel in such conditions. On the contrary, at $120^{\circ} \mathrm{C} / \mathrm{min}$, the porosity is higher in the membrane thickness and the water sorption is much lower, what confirms that the chemical cross-linking was more efficient at $120^{\circ} \mathrm{C} / \mathrm{min}$ that at $2{ }^{\circ} \mathrm{C} / \mathrm{min}$, probably since the chemical reaction is favored at high temperature and even if the target temperature $\left(60^{\circ} \mathrm{C}\right)$ was the same for both conditions, it was reached earlier at $120^{\circ} \mathrm{C} / \mathrm{min}$ than at $2^{\circ} \mathrm{C} / \mathrm{min}$, i.e. $40 \mathrm{~s}$ and $1200 \mathrm{~s}$, respectively.

\subsubsection{Mechanical properties}

First, the influence of the casting speed was investigated on the mechanical properties of HPC membranes. Table 4 reports the tensile strength, the maximum elongation and the Young modulus for two casting speeds, i.e. 3.4 and $15.0 \mathrm{~cm} / \mathrm{s}$.

Table 4. Influence of casting speed on the mechanical properties of HPC membranes (HPC $20 \%$, GA $5 \%, \mathrm{HCl} 1 \%)$ prepared by LCST-TIPS method at $60^{\circ} \mathrm{C}$.

\begin{tabular}{c|c|c|c|c|}
\multicolumn{2}{c|}{} & $\begin{array}{c}\text { Tensile strength } \\
(\mathrm{MPa})\end{array}$ & $\begin{array}{c}\text { Maximum } \\
\text { elongation (\%) }\end{array}$ & $\begin{array}{c}\text { Young module } \\
\text { (Mpa) }\end{array}$ \\
\hline \multirow{2}{*}{ Casting speed } & $3.4 \pm 0.2 \mathrm{~cm} / \mathrm{s}$ & $1.46 \pm 0.2$ & $1.00 \pm 0.1$ & $1173 \pm 100$ \\
\cline { 2 - 5 } & $15.0 \pm 0.5 \mathrm{~cm} / \mathrm{s}$ & $3.34 \pm 0.2$ & $2.15 \pm 0.1$ & $1011 \pm 100$ \\
\hline
\end{tabular}

These results clearly exhibit that the mechanical properties of HPC membranes cast with the higher shear rate were improved compared to those of membranes prepared with the lower one. Rheological conditions during casting and spinning have been proved to be an important parameter influencing the final membrane performance in the case of dense and porous active layer, as well 
[36-37]. It is assumed that high shear rate promotes the polymer chain orientation resulting in their closer packing in the bulk matrix. This effect can account for our findings regarding the improvement of mechanical properties. Despite the modest range of casting speed rate studied, a significant increase of tensile strength and elongation at break was obtained. It can then be expected that even much better properties should be reached in fabrication of HPC hollow fibers by spinning in which high shear rates are usually applied. For the rest of this paper, only data obtained with membranes prepared using the maximum casting speed attainable with our set-up will be presented.

The influence of the temperature ramp and of the formulation on the mechanical properties was also investigated and the results are reported in Table 5. As mentioned in the section 2.2.4.4, three successful measurements were used to obtain average values for mechanical properties. For each condition, one characteristic graph representing the tensile stress versus the tensile strain was reported in Fig. 5.

Table 5. Influence of the temperature ramp on the mechanical properties of HPC membranes (HPC $20 \%, \mathrm{GA} 5 \%, \mathrm{HCl} 1 \%)$ prepared by LCST-TIPS method at $60^{\circ} \mathrm{C}$

Temperature ramp

\begin{tabular}{c|c|c|c|}
\cline { 3 - 3 } \multicolumn{2}{c|}{} & $\mathbf{1 2 0}{ }^{\circ} \mathrm{C} / \mathrm{min}$ & $\mathbf{2}{ }^{\circ} \mathrm{C} / \mathbf{m i n}$ \\
\hline \multirow{3}{*}{ Membranes HPC/GA } & Tensile strength (MPa) & $3.34 \pm 0.2$ & $2.80 \pm 0.2$ \\
\cline { 2 - 4 } & Maximum elongation (\%) & $2.15 \pm 0.1$ & $2.06 \pm 0.1$ \\
\cline { 2 - 4 } & Young module (MPa) & $1011 \pm 200$ & $1083 \pm 200$ \\
\hline \multirow{3}{*}{ Membranes HPC/GA/PEG200 } & Tensile strength (MPa) & $1.7 \pm 0.2$ & $2.00 \pm 0.2$ \\
\cline { 2 - 4 } & Maximum elongation (\%) & $0.46 \pm 0.1$ & $0.46 \pm 0.1$ \\
\cline { 2 - 4 } & & & $651 \pm 100$ \\
\hline
\end{tabular}




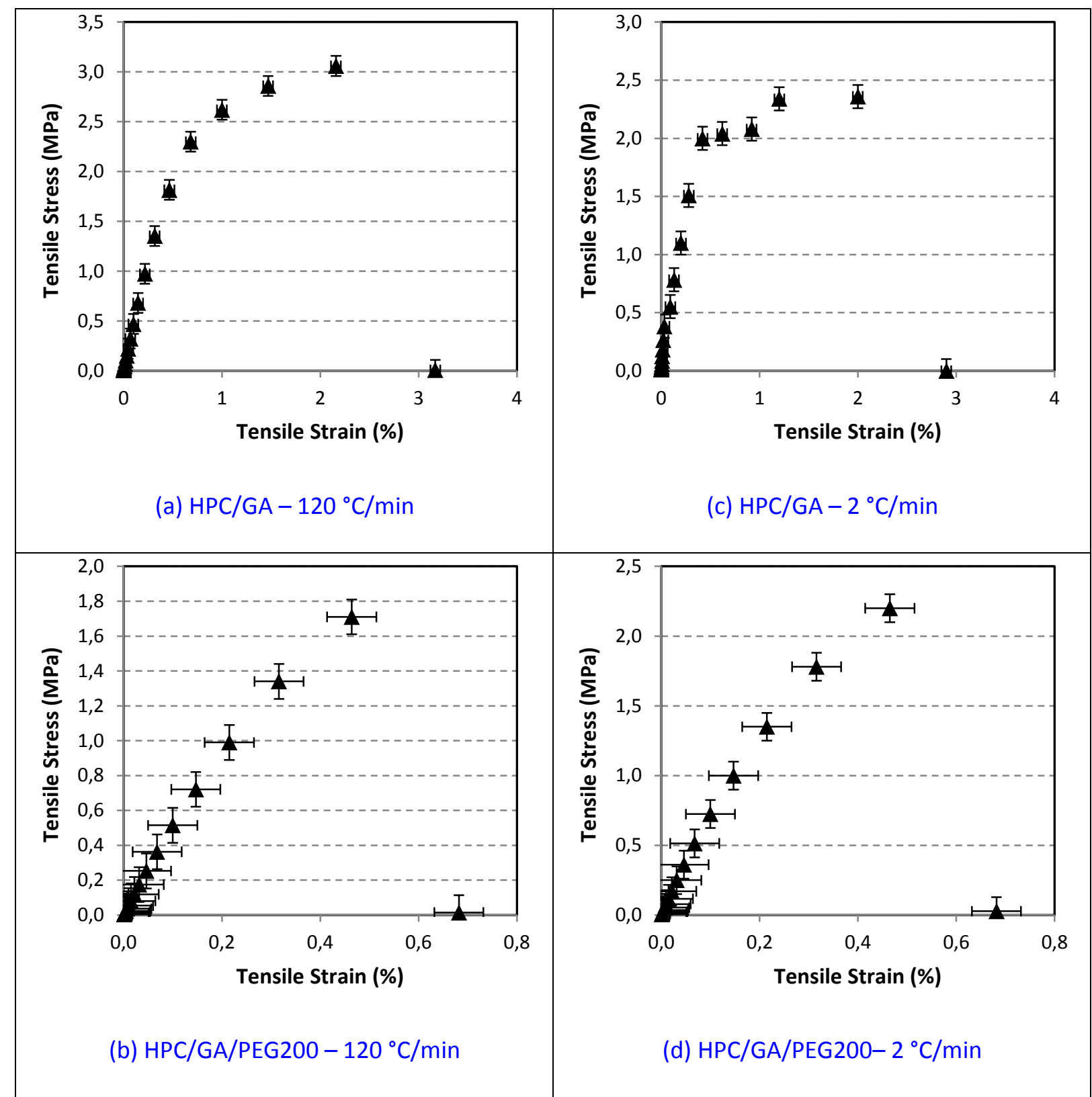

Figure 5. Tensile stress versus tensile strain corresponding to the mechanical properties presented in Table 5.

As can be seen, whatever the formulation, the temperature ramp did not show any drastic effect on the mechanical properties. It was concluded that the polymer chain arrangement seems not to be disturbed too much by the crosslinking at surface or at depth. It should be noted that an expected decreasing trend was observed in the latter case. By contrast, the presence of a porogen (PEG200) in the initial formulation strongly affected the whole mechanical properties. The elongation at break markedly decreased indicating a likely anti-plasticizer effect of PEG200. Although the drop of both 
the tensile strength and Young modulus is not in agreement with this assumption, our findings consistent with previous report showing that extruded films made of HPC and 1\% PEG400 exhibited instability of mechanical properties with a continuous decrease of elongation at break and tensile strength during storage [38].

At this point, the obtained mechanical properties were not high enough to enable experiments under pressure. For this reason, the HPC polymer dope solutions were cast onto a non-woven PET support prior to further filtration. SEM cross-section of supported membranes is presented in Fig. 6, exhibiting a uniform HPC layer that did not clog the non-woven porosity.

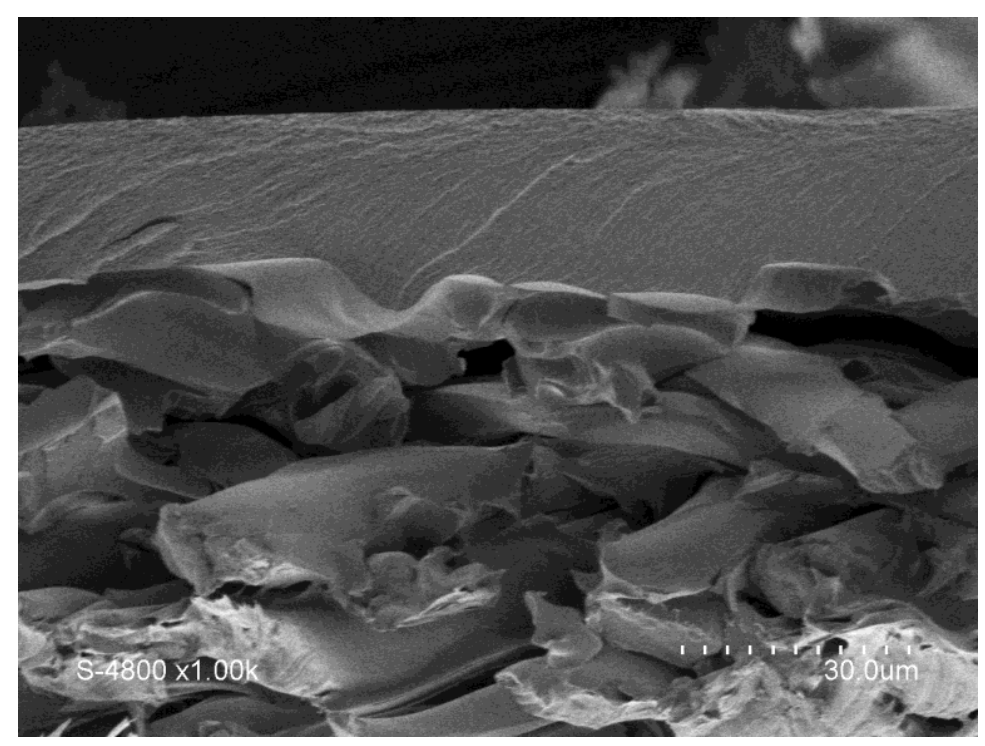

Figure 6. Cross-section of HPC membrane onto a non-woven PET support

\subsubsection{Permeability results}

The procedure for the filtration tests was detailed in the material and methods section. A membrane conditioning precedes the filtration tests, corresponding to a gradual increase of pressure from 0 to 3.5 bar each 0.2 bar.

The filtration tests were conducted in this work for different kinds of membranes, i.e. different formulations and different preparation procedures: membranes with or without PEG200 were studied; two temperature ramps were tested $\left(2\right.$ and $\left.120^{\circ} \mathrm{C} / \mathrm{min}\right)$ during the membrane preparation 
by LCST-TIPS process in the Linkam hot stage; for the highest temperature ramp, membranes were prepared under $50 \%$ and $100 \% \mathrm{RH}$. The results of filtration measurements were reported in Table 6.

Table 6. Permeability of HPC membranes for two temperature ramps (HPC $20 \mathrm{wt} \%, \mathrm{GA} 5 \mathrm{wt} \%, \mathrm{HCl}$ $1 \mathrm{wt} \%$ ), under $50 \%$ and $100 \% \mathrm{RH}$, with or without porogen agent (PEG200).

\section{Temperature ramp}

\begin{tabular}{|c|c|c|c|}
\cline { 2 - 4 } \multicolumn{1}{c|}{} & $120{ }^{\circ} \mathrm{C} / \mathrm{min}(\mathrm{RH} 50 \%)$ & $2{ }^{\circ} \mathrm{C} / \mathrm{min}(\mathrm{RH} 50 \%)$ & $120{ }^{\circ} \mathrm{C} / \mathrm{min}(\mathrm{RH} 100 \%)$ \\
\hline Membrane HPC/GA & $0 \mathrm{~L} / \mathrm{h} / \mathrm{m}^{2} / \mathrm{bar}$ & $0 \mathrm{~L} / \mathrm{h} / \mathrm{m}^{2} / \mathrm{bar}$ & $0 \mathrm{~L} / \mathrm{h} / \mathrm{m}^{2} / \mathrm{bar}$ \\
\hline Membrane HPC/GA/PEG & $12.2 \mathrm{~L} / \mathrm{h} / \mathrm{m}^{2} / \mathrm{bar}$ & $1 \mathrm{~L} / \mathrm{h} / \mathrm{m}^{2} / \mathrm{bar}$ & $1 \mathrm{~L} / \mathrm{h} / \mathrm{m}^{2} / \mathrm{bar}$ \\
\hline
\end{tabular}

The whole membranes prepared without PEG200 were almost not permeable to water, even for the highest pressures applied and whatever the preparation procedure. By contrast, adding the porogen agent in the initial formulation helped opening the membrane porosity, and in this case the filtration performances were significantly affected by the preparation procedure: at $2^{\circ} \mathrm{C} / \mathrm{min}$ the membrane permeability was very low and the flux was almost null until 3 bars. At 3 bars, a flux around only $3 \pm 0,2 \mathrm{~L} / \mathrm{h} / \mathrm{m}^{2}$ was detected. By contrast, the membranes prepared with PEG200 under $50 \% \mathrm{RH}$ at $120^{\circ} \mathrm{C} / \mathrm{min}$, were permeable. The water filtration flux was around $38 \pm 5 \mathrm{~L} / \mathrm{h} / \mathrm{m}^{2}$ at 3 bars in these conditions. This result clearly indicates that the membrane preparation procedure had a significant effect on the membrane permeability, suggesting that the membrane morphology could have been also affected by the membrane formation procedure. The Figure 7 represents the flux versus the pressure for the membranes obtained with PEG200 under $50 \% \mathrm{RH}$ at $120^{\circ} \mathrm{C} / \mathrm{min}$. 


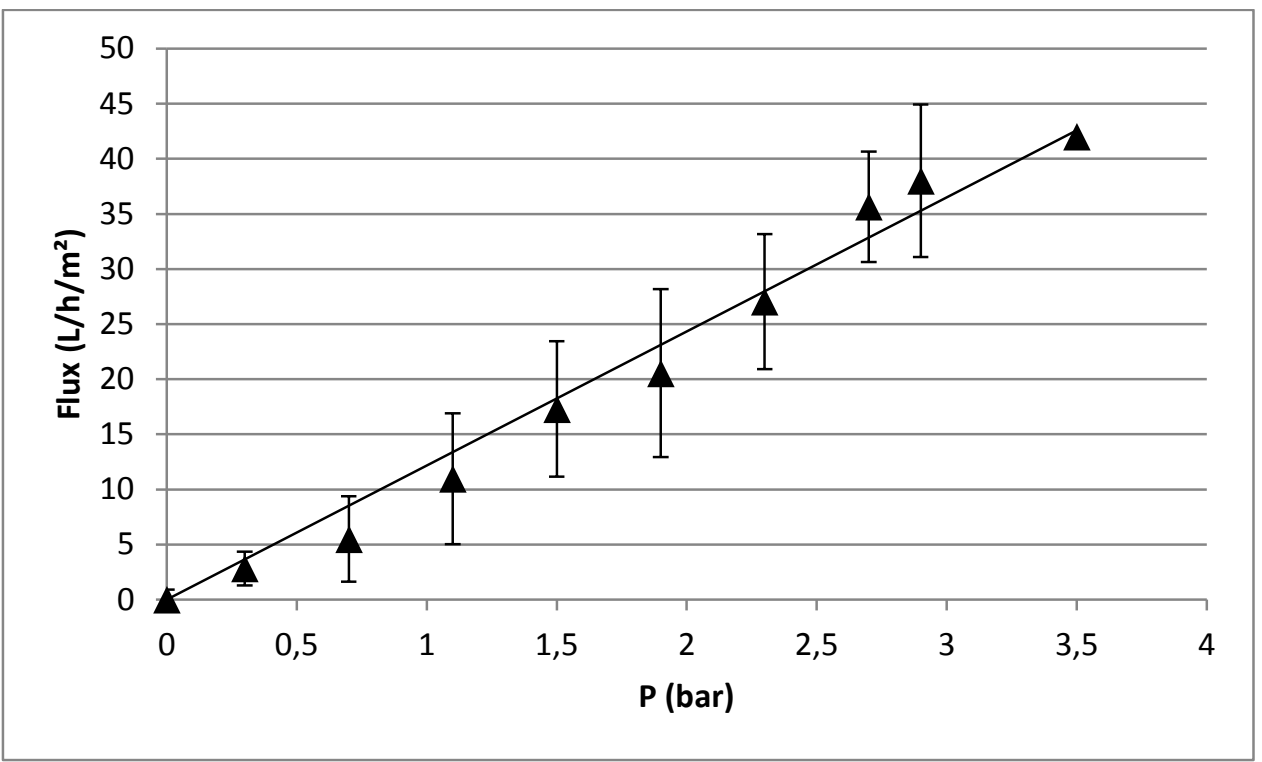

Figure 7. Flux versus the pressure for the membranes obtained with PEG200 under $50 \%$ RH at $120^{\circ} \mathrm{C} / \mathrm{min}$.

At $2{ }^{\circ} \mathrm{C} / \mathrm{min}$, the previous results of water sorption exhibited that the water intake within the membrane is high comparing to $120^{\circ} \mathrm{C} / \mathrm{min}$, suggesting that the membrane could be assimilated to a hydrogel. Such a behavior was related to a poor cross-linking at $2{ }^{\circ} \mathrm{C} / \mathrm{min}$ leading to the formation of a hydrogel, allowing water sorption in large amount when immersed in water. The SEM photographs pointed out that the membrane structure was denser at $2{ }^{\circ} \mathrm{C} / \mathrm{min}$ than at $120^{\circ} \mathrm{C} / \mathrm{min}$, in agreement with the permeability results, and they also exhibited that the filtration layer was located in the middle of the membrane, especially at $2{ }^{\circ} \mathrm{C} / \mathrm{min}$. In this region, the membrane structure is almost dense, explaining the poor filtration performances of the membranes prepared in these conditions.

On the contrary, at $120^{\circ} \mathrm{C} / \mathrm{min}$, the chemical cross-linking is more efficient because the final temperature is reached after only $20 \mathrm{~s}$. Thus, the characteristic length between cross-linking bonds is lower than at $2^{\circ} \mathrm{C} / \mathrm{min}$ and the water sorption is significantly reduced. 


\section{Conclusion}

This paper deals with the development of a novel porous polymeric membrane from biobased polymer (HPC) and without the use of organic solvent. A thermally induced phase separation process was used to induce the phase inversion, but increasing the temperature above the LCST of $\mathrm{HPC} /$ water solution. Using optical transmission methods, the phase diagram of the polymeric system was investigated, and the cloud point curve was plotted in the polymer concentration range [1$30 \mathrm{wt} \%]$. The spinodal curve was determined in the same time and it was demonstrated that the metastable region is very thin in the classical concentration range used to make membranes (1025 wt\% of polymer). SEM photographs exhibited that HPC membranes were porous in the whole cross-section, characterized by a quite low porosity, especially when the temperature ramp is low $\left(2{ }^{\circ} \mathrm{C} / \mathrm{min}\right)$. At such a low temperature ramp, it was suggested that the chemical crosslinking reaction using GA was delayed thus forming a hydrogel matrix. At higher temperature ramp $\left(120^{\circ} \mathrm{C} / \mathrm{min}\right)$, the membrane showed lower swelling and better filtration performances. The prove of concept was therefore demonstrated: a membrane, stable in water, was made from water-soluble biobased polymer, which may reduce the use of non-renewable resource materials (petroleum) and reduce pollution levels, in agreement with the principles of green chemistry.

\section{Acknowledgment}

The authors would like to address acknowledgments to the French National Agency of Research (ANR) which supported this study through the project POMEWISO (ANR-10-CD2I-0012). H.G. also thanks postdoctoral fellowship from CEA-Enhanced Eurotalents program, cofunded by FP7 Marie Skłodowska-Curie COFUND Program, grant agreement Nº 600382. 


\section{References}

[1] M. Ulbricht, Advanced functional polymer membranes, Polymer 47 (2006) 2217-2262.

[2] F. Liu, N. A. Hashim, Y. Liu, M.R. Moghareh Abed, K. Li, Progress in the production and modification of PVDF membranes, J. Membr. Sci. 375 (2011) 1-27.

[3] M. Mulder, Basic principles of membrane technology, Kluwer Academic Publishers, 1991

[4] Z.Y. Cui, C.H. Du, Y.Y. Xu, G.L. Ji, B.K. Zhu, Preparation of porous PVDF membrane via thermally induced phase separation using sulfolane, Journal of Applied Polymer Science, 108 (2008) 272-280.

[5] D.R. Lloyd, S.S. Kim and K.E. Kinzerb, Microporous membrane formation via thermally induced phase separation. II. Liquid-liquid phase separation, J. Membr. Sci, 64 (1991) 1-11.

[6] H. Matsuyama, M. Yuasa, Y. Kitamura, M. Teramoto, D.R. Lloyd, Structure control of anisotropic and asymmetric polypropylene membrane prepared by thermally induced phase separation, J. Membr. Sci, 179 (2000) 91-100.

[7] S. Rajabzadeh, T. Maruyama, T. Sotani, H. Matsuyama, Preparation of PVDF hollow fiber membrane from a ternary polymer/solvent/nonsolvent system via thermally induced phase separation (TIPS) method, Separation and Purification Technology 63 (2008) 415-423.

[8] P. Atkinson, D. R. Lloyd, Anisotropic flat sheet membrane formation via TIPS: thermal effects, J. Membr. Sci. 171 (2000) 1-18

[9] P. Atkinson, D. R. Lloyd, Anisotropic flat sheet membrane formation via TIPS: atmospheric convection and polymer molecular weight effects, J. Membr. Sci. 175 (2000) 225-238

[10] H. Matsuyama, S. Berghmans, M. T. Batarseh, D. R. Lloyd, Effects of thermal history on anisotropic and asymmetric membranes formed by thermal induced phase separation, Membr. Sci. $142(1998) 27-42$

[11] H. Matsuyama, S. Berghmans, D. R. Lloyd, Formation of hydrophilic microporous membranes via thermally induced phase separation, Membr. Sci. 142 (1998) 213-224

[12] A. Figoli, T. Marino, S. Simone, E. Di Nicolò, X.-M. Li, T. He, S. Tornaghi, E. Drioli, Towards nontoxic solvents for membrane preparation: a review, Green Chem., 16 (2014) 4034-4059 
[13] X.L. Li, L.P. Zhu, B.K. Zhu, Y.Y. Xu, High-flux and anti-fouling cellulose nanofiltration membranes prepared via phase inversion with ionic liquid as solvent, Separation and Purification Technology 83 (2011) 66-73.

[14] M. Bodzek, K. Konieczny, Comparison of various membrane types and module configurations in the treatment of natural water by means of low-pressure membrane methods, Separation and Purification Technology 14 (1998) 69-78

[15] W.Y. Chuang, T.H. Young, W.Y. Chiu, The effect of acetic acid on the structure and filtration properties of poly(vinyl alcohol) membranes, J. Membr. Sci. 172 (2000) 241-251.

[16] O. M’barki, A. Hanafia, D. Bouyer, C. Faur, R. Sescousse, U. Delabre, C. Blot, P. Guenoun, A. Deratani, D. Quemener, C. Pochat-Bohatier, Greener method to prepare porous polymer membranes by combining thermally induced phase separation and crosslinking of poly(vinylalcohol) in water, J. Membr. Sci. 458 (2014) 225-235.

[17] F. Wang, S. Saeki, T. Yamaguchi, Absolute prediction of upper and lower critical solution temperatures in polymer/solvent systems based on corresponding state theory, Polymer 40 (1999) $2779-2785$.

[18] J.J. Qin, M.H. Oo, Y. Li, Development of high flux polyethersulfone hollow fiber ultrafiltration membranes from a low critical solution temperature dope via hypochlorite treatment, J. Membr. Sci. 247 (2005) 137-142.

[19] J.J. Qin, F.S. Wong, Y. Li, Y.T. Liu, A high flux ultrafiltration membrane spun from PSU/PVP (K90)/DMF/1,2-propanediol, J. Membr. Sci. 211 (2003) 139-147.

[20] J.J. Qin, M.H. Oo, Y.M. Cao, L.S. Lee, Development of a LCST membrane forming system for cellulose acetate ultrafiltration hollow fiber, Separation and Purification Technology 42 (2005) 291295.

[21] E.K. Just, T.G. Majewicz, Cellulose ethers, in "Encyclopedia of Polymer Science and Engineering". H.F. Mark, N.M. Bikales, C.G. Overberger and G. Menges, (Eds.) John Wiley and Sons, New York, (1985),3-226. 
[22] R. Gottlieb, C. Kaiser, U. Gohs, K.F. Arndt, Temperature sensitive hydrogels based on hydroxypropylcellulose by high energy irradiation. Macromolecular Symposia, 254 (2007) 361-369.

[23] E. Marsano, E. Bianchi, L. Sciutto, Microporous thermally sensitive hydrogels based on hydroxypropylcellulose crosslinked with poly-ethyleneglicol diglycidyl ether. Polymer, 44 (2003) 6835-6841.

[24] S.G. Hirsch, R.J. Spontak, Temperature-dependent property development in hydrogels derived from hydroxypropylcellulose. Polymer, 43 (2002) 123-129.

[25] T. Kyu, P. Zhuang, P. Mukherjee, Phase-Separation Dynamics of Aqueous Hydroxypropyl Cellulose Solutions, ACS Symposium Series, 384 (1989) 266-283.

[26] E. Marsano, G. Fossati, Phase diagram of water soluble semirigid polymers as a function of chain hydrophobicity, Polymer Communication, 41 (2000) 4357-4360.

[27] R.S. Werbowyj, D.G. Gray, Ordered Phase Formation in Concentrated Hydroxpropylcellulose Solutions, Macromolecules, 13, (1980) 69-73.

[28] F.M. Winnik, Effect of temperature on aqueous solutions of pyrene-labeled (hydroxypropyl)cellulose, Macromolecules, 20 (1987) 2745-2750

[29] T. Kyu and P. Mukherjee, Kinetics of Phase Separation by Spinodal Decomposition in a Liquid Crystalline Polymer Solution, Liq. Cryst.[, 3 (1988) 631-644

[30] S.-P. Rwei, M.-S. Lyu, 3-D phase diagram of $\mathrm{HPC} / \mathrm{H}_{2} \mathrm{O} / \mathrm{H}_{3} \mathrm{PO}_{4}$ tertiary system, Cellulose, 19 (2012) 1065-1074

[31] S. Fujii, NA. Sasaki, M. Nakata, Rheological Studies on the Phase Separation of Hydroxypropylcellulose Solution Systems, J. of Poly. Sci.: Part B: Pol. Physics, 39 (2001) 1976-1986

[32] R.S. Werbowyj, D.G. Gray, Liquid crystalline structure in aqueous hydroxypropyl cellulose solutions , Mol. Cryst. Liq. Cryst., 34 (1976), 97-103

[33] Koningsveld R, Stockmayer WH, Nies E Polymer phase diagrams. A textbook. Oxford University Press, Oxford, New York (2001) 140 
[34] Poly(N-isopropylacrylamide) Phase Diagrams: Fifty Years of Research, A. Halperin, M. Kröger and F. M. Winnik, Angew. Chem., Int. Ed., 54 (2015) 15342-15367

[35] S. Suto, Hydroxypropylcellulose (Crosslinked, Cholesteric Liquid Crystal), Polymeric materials encyclopedia, Vol 5 (1996)

[36] T.S Chung, S.K Teoh, W.W.Y Lau, M.P Srinivasan, Effect of shear stress within the spinneret on hollow fiber membrane morphology and separation performance, Ind. Eng. Chem. Res., 37 (1998) 3930-3938

[37] A.F. Ismail, A.R. Hassan, Formation and characterization of asymmetric nanofiltration membrane: Effect of shear rate and polymer concentration, J. Membr. Sci. 270, (2006) 57-72

[38] M. A. Repka, T. G. Gerding, S. L. Repka, J. W. McGinity, Influence of Plasticizers and Drugs on the Physical-Mechanical Properties of Hydroxypropylcellulose Films Prepared by Hot Melt Extrusion, Drug Development and Industrial Pharmacy, 25(5) (1999) 625-633 